Ophthalmologe 2022 $119: 737-740$

https://doi.org/10.1007/s00347-021-01426-0

Eingegangen: 7. April 2021

Angenommen: 10. Mai 2021

Online publiziert: 9. Juni 2021

๑) Der/die Autor(en) 2021

\author{
Bettina Klieme ${ }^{1} \cdot$ Arne Viestenz $^{1} \cdot$ Stephan Eisenmann ${ }^{2} \cdot$ Ricarda Wienrich $^{1}$ \\ 'Klinik und Poliklinik für Augenheilkunde, Martin-Luther-Universität Halle-Wittenberg, Halle (Saale), \\ Deutschland \\ ${ }^{2}$ Klinik und Poliklinik für Innere Medizin I (Pneumologie), Martin-Luther-Universität Halle-Wittenberg, \\ Halle (Saale), Deutschland
}

\title{
Akuter progredienter Visusverlust als Erstmanifestation einer karzinomassoziierten Retinopathie (CAR) bei neuroendokrinem kleinzelligem Lungenkarzinom
}

\section{Falldarstellung}

\section{Anamnese}

Notfallmäßig stellte sich ein 60-jähriger Patient mit beidseitigem schmerzlosem Visusabfall seit 10 Tagen vor. Anamnestisch bestand 6 Wochen zuvor ein grippaler Infekt, seitdem war er abgeschlagen. Weitere B-Symptomatik wurde verneint, lediglich unter körperlicher Belastung würden Gelenk- und Rückenschmerzen auftreten, ein Zeckenbiss sei für 1998 erinnerlich. Anamnestisch habe der Patient 2014 eine transitorisch ischämische Attacke erlitten und werde aufgrund einer Hypercholesterinämie medikamentös behandelt. Die Nikotinanamnese ergab 40 pack years.

\section{Klinischer Befund}

Der initiale Visus betrug rechts 0,2 und links 0,16. Der Augeninnendruck war normoton. Im Vorderabschnitt fanden sich beidseits eine Bindehauthyperämie sowie pigmentierte Endothelbeschläge, Isokorie bei träger Pupillenreaktion. Fundoskopisch bestanden inferior ausgeprägte Glaskörpertrübungen, die retinalen Gefäße waren verdünnt und wiesen periphere Einscheidungen auf (• Abb. 1). In der optischen Kohärenz-Tomographie
(OCT) war eine Destruktion der retinalen Pigmentepithel- und Photorezeptorschicht nachweisbar (• Abb. 2 und 3). Fluoreszenzangiographisch zeigte sich eine deutliche Gefäßrarefizierung mit choroidalen Füllungsdefekten (• Abb. 4).

\section{Diagnose}

Karzinomassoziierte Retinopathie(CAR).

\section{Therapie und Verlauf}

Bei initial bestehendem Verdacht auf eine infektiöse Genese erfolgte unter antibiotischer Abschirmung (Cefuroxim $2 \mathrm{~g}$ intravenös) eine systemische hoch dosierte Prednisolon-Gabe (initial $100 \mathrm{mg}$ intravenös, im Verlauf Steigerung auf $250 \mathrm{mg}$, dann ausschleichend). Die serologischen Untersuchungen (TPHA, TBC, HIV, HSV, VZV, CMV, Borrelien, ANA, ANCA, Differenzialblutbild, ACE, IL2-Rezeptor) waren unauffällig. Der Varizella-Zoster-Virus- und Borrelientiter zeigten sich grenzwertig erhöht, sodass die Therapie um intravenöses Aciclovir ergänzt wurde. Der Liquorbefund, die kraniale Magnetresonanztomographie und die Thoraxröntgenuntersuchung waren ebenfalls unauffällig. Da sich der Befund trotz oben genannter Therapie rasch progredient verschlechterte, wurde eine diagnostische Vitrektomie durchgeführt, in der sich kein Hinweis auf ein intraokuläres Lymphom oder eine Infektion fand. Am 13. Behandlungstag wurde der Recoverin-Antikörper nachgewiesen. Bei noch unklarem Primarius und sich verschlechterndem Befund wurde als Therapieversuch eine Plasmapherese durchgeführt. Das Tumorscreening (Abdomensonographie, Gastroskopie, Computertomographie des Thorax) erbrachte bei sonst unauffälligem Befund einen isolierten paratrachealen Lymphknoten, der auf $2,4 \mathrm{~cm}$ vergrößert war (- Abb. 5).

Mittels Feinnadelaspiration ließ sich ein kleinzelliges neuroendokrines Lungenkarzinom sichern (T1a, N2, M0, Stadium III A), eine Metastasierung fand sich in der Positronen-EmissionsTomographie/Computertomographie (PET-CT) nicht. Es wurde eine kurative Radiotherapie (44Gy) mit simultaner Chemotherapie (6 Zyklen mit Carboplatin/Etoposid/Atezolizumab) durchgeführt. Als dauerhafte Immuntherapie erhält der Patient Atezolizumab. Unter dieser Therapie zeigt sich bis zum aktuellen Zeitpunkt (1 Jahr nach Erstdiagnose) eine gute partielle Remission des Tumors. Die Sehschärfe regenerierte nicht und blieb beidseits bei Handbewegungen. 


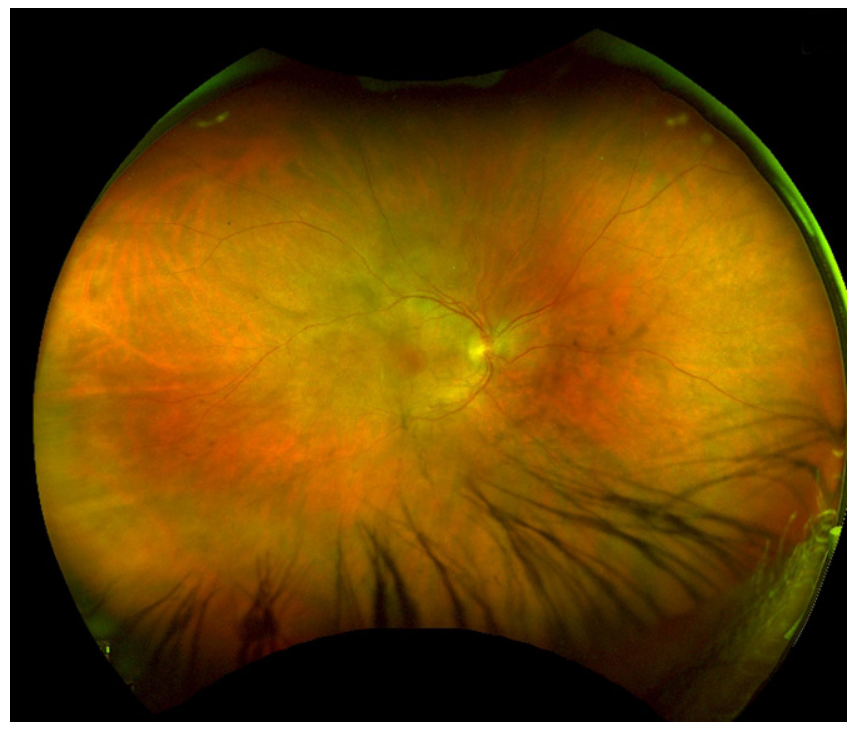

Abb. $1 \varangle$ Initiales Fundusfoto mit verdünnten retinalen Gefäßen mit peripheren Einscheidungen
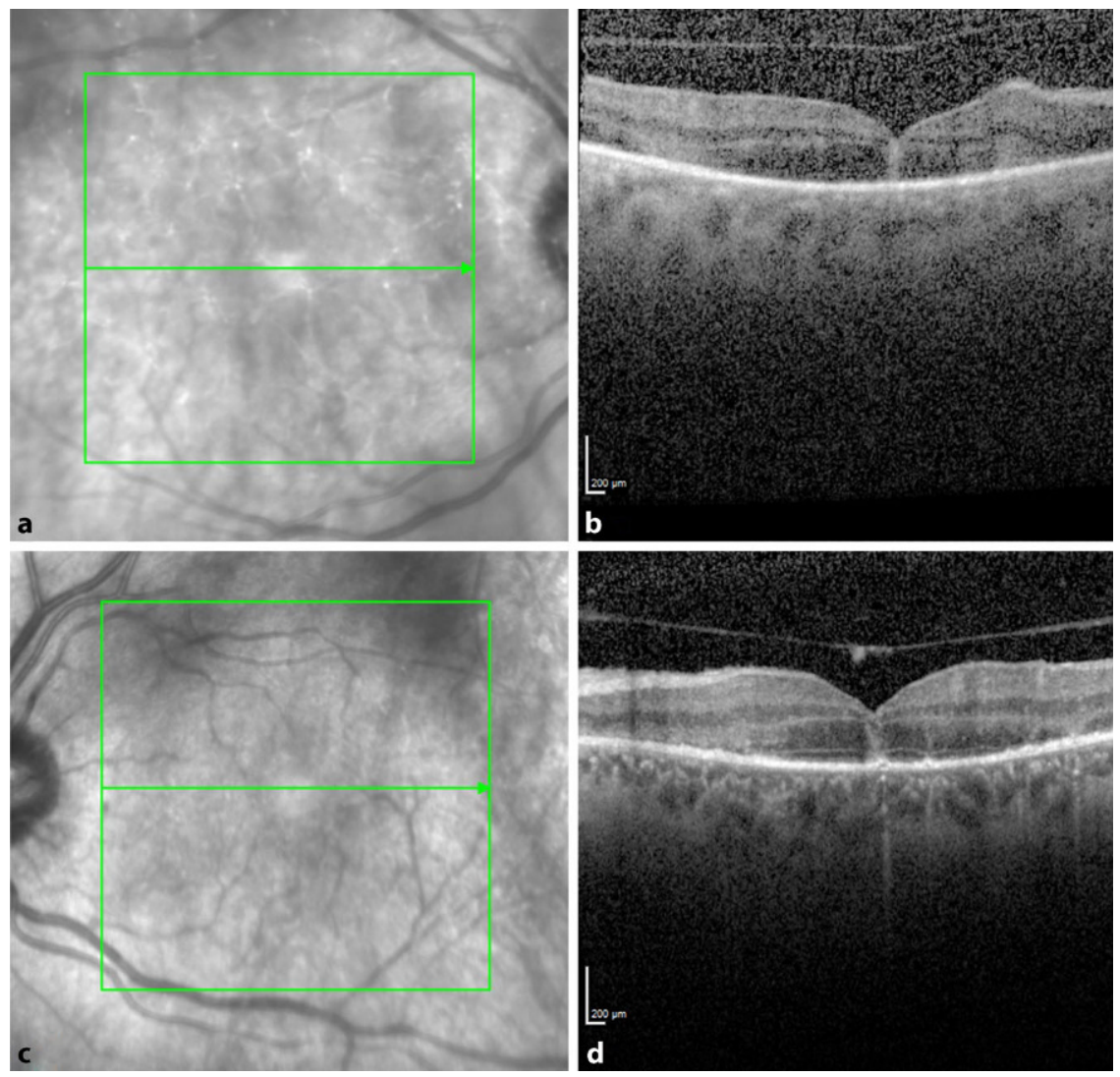

Abb. 2 \& Initiale OCT mit Glaskörpertrübungen und nach zentral fortgeschrittener Destruktion der äußeren Netzhautschichtung am rechten $(\mathbf{a}, \mathbf{b})$ und linken Auge $(\mathbf{c}, \mathbf{d})$

\section{Diskussion}

Lungenkarzinome sind mit $24 \%$ die häufigste Krebstodesursache bei Männern und die zweithäufigste bei Frauen. Das mittlere Erkrankungsalter liegt bei ca. 70 Jahren. Typische Symptome sind
Husten, Gewichtsverlust, Luftnot und Brustschmerzen [4].

Kleinzellige neuroendokrine Lungenkarzinome machen ca. $15 \%$ aller Bronchialtumoren aus und werden histopathologisch der Obergruppe der epithelialen Tumoren und der Subgruppe der neuroendokrinen Tumoren zuge- ordnet. Untherapiert beträgt die mittlere Überlebenszeit wenige Monaten, kann aber durch gute Therapiesensibilität auf 8 bis 20 Monate erhöht werden. Die 5-Jahres-Überlebensrate liegt bei 10-20\%, bei Auftreten von Metastasen sinkt sie auf weniger als $1 \%$. Die Therapie besteht in einer kombinierten Chemotherapie, welche mittlerweile standardisiert mit Checkpointinhibitoren zur Immunmodulation ergänzt wird. Je nach Stadium und Lokalisation kann eine Bestrahlung bzw. operative Intervention erfolgen.

Etwa $10 \%$ der Patienten mit Lungenkarzinom entwickeln ein paraneoplastisches Syndrom, hierzu zählt auch die karzinomassoziierte Retinopathie (CAR) [4].

Die CAR wurde erstmalig 1976 beschrieben und kann bei verschiedenen Tumorerkrankungen auftreten [5]. Am häufigsten ist sie im Zusammenhang mit Lungen- und Mammakarzinomen beschrieben $[5,7]$. Es handelt sich um ein seltenes Krankheitsbild, zu dem bisher nur kleine Fallserien bzw. Einzelfallbeschreibungen vorliegen, die Inzidenz ist unbekannt [7].

Als ursächlich wird eine Immunreaktion gegen den Tumor angenommen, die zu einer Autoantikörper-vermittelten Zerstörung verschiedener Proteine, wie z. B. dem Recoverin, führt. Recoverin (23-kd-Antigen) kommt in den Photorezeptoren der Retina, dem Nervus opticus und der Epiphyse vor und steuert einen Kalziumkanal [3, 5, 7]. Wird es im Rahmen der Immunreaktion zerstört, führt es zur Zytolyse der Photorezeptoren [6]. Dies verursacht die klassischen Symptome einer CAR mit Photopsien, Blendempfindlichkeit, Gesichtsfeldeinengung und Visusverlust. Recoverin ist nicht spezifisch oder sensitiv für eine CAR und kann beispielsweise auch bei Patienten mit Retinopathia pigmentosa vorkommen. Antiretinale Antikörper wurden auch bei gesunden Kontrollgruppen und zahlreichen weiteren systemischen und retinalen Erkrankungen gefunden $[1,7]$.

Der Beginn der okulären Symptome kann zeitlich deutlich vor Entdeckung des Primärtumors liegen und sich über Monate bis Jahre erstrecken $[5,7]$. 


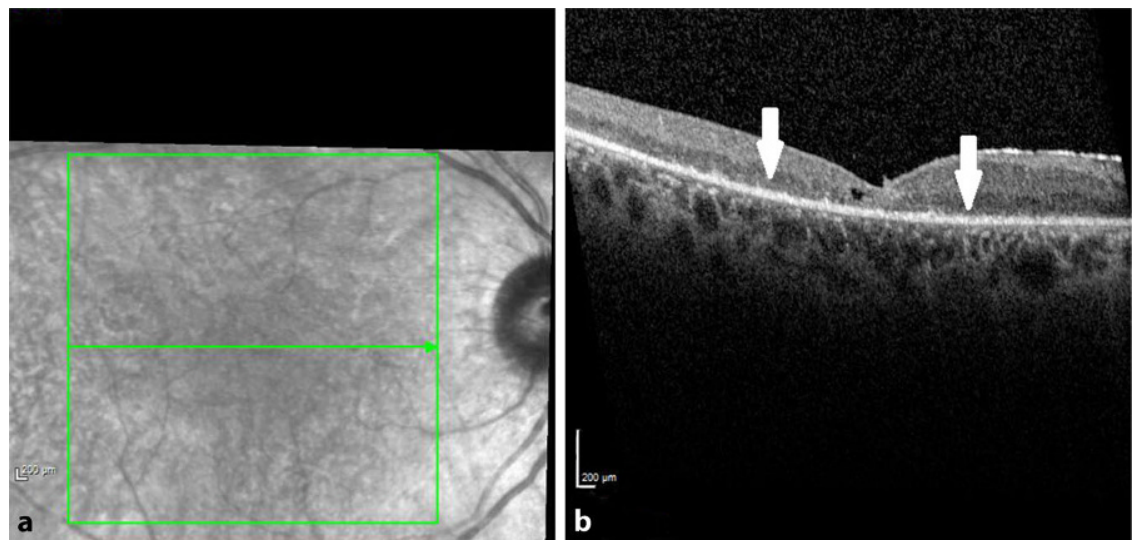

Abb. $3 \Delta$ Verlaufs-OCT mit progredienter Verdünnung der äußeren Netzhautschichtung (Pfeile)

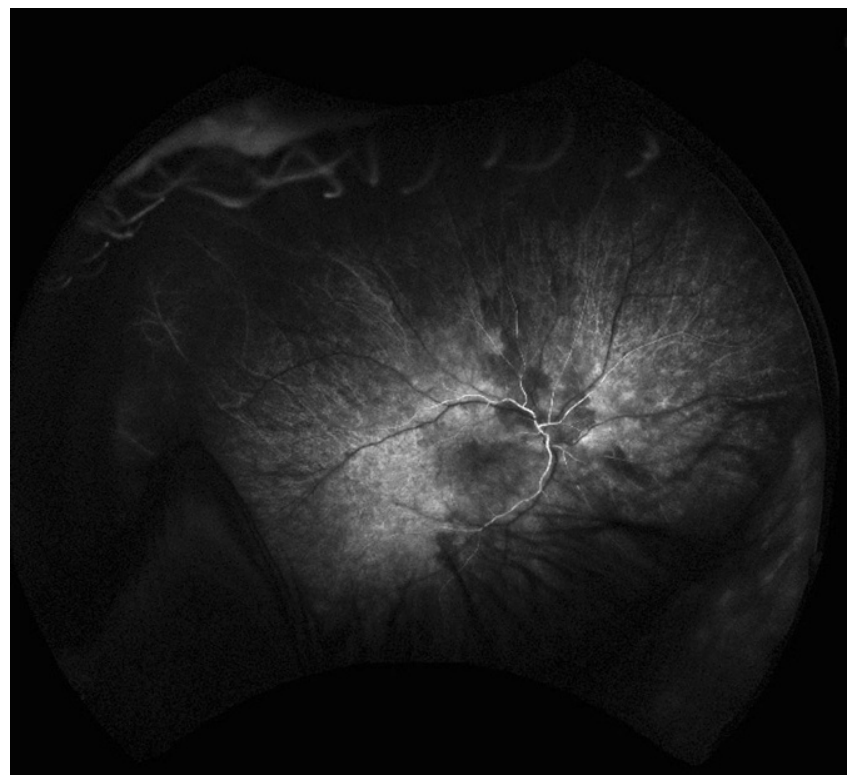

Abb. $4<$ Fluoreszenzangiographie mit Gefäßrarefizierung und choroidalen Füllungsdefekten

In den Frühformen der CAR können retinale Auffälligkeiten fehlen. Im Verlauf werden meist verdünnte Arteriolen, fleckiges retinales Pigmentepithel, abgeblasste Papillen, Glaskörperzellen, arterioläre Ummantelung und eine Periphlebitis beobachtet $[1,5,7]$. Das Elektroretinogramm zeigt eine deutlich abgeschwächte oder fehlende photopische und skotopische Reaktion [2, 5, 7]. In der OCT zeigen sich ein Verlust der äußeren retinalen Schichten sowie zystische Hohlräume und schisisartige Veränderungen [2, 3, 5].

Neben dem Recoverin können weitere Antigene (z. B. Alpha-Enolase, Transducin), die von verschiedenen retinalen Strukturen exprimiert werden, zu einer CAR führen $[1,7]$.
Ein standardisiertes diagnostisches Vorgehen zum Nachweis der Antikörper (ELISA, Western Blot, Immunhistochemie) sowie eine eindeutige Zuordnung des prädiktiven Wertes bei positiven Testergebnissen sind aktuell noch nicht vorliegend [1].

Ein etabliertes therapeutisches Vorgehen bei CAR existiert ebenfalls bisher noch nicht. Basis sind verschiedene immunsupprimierende Therapien. Deren Effekt auf die visuelle Rehabilitation ist stark davon abhängig, inwieweit bereits bei Therapiebeginn die irreversible Zytolyse der Photorezeptoren fortgeschritten ist $[3,5,7]$.

Neben einer systemischen Steroidgabe sind lokale Applikationen (subtenonal oder intravitreal) möglich. Ergänzend ist eine Immunsuppression mit Azathio-

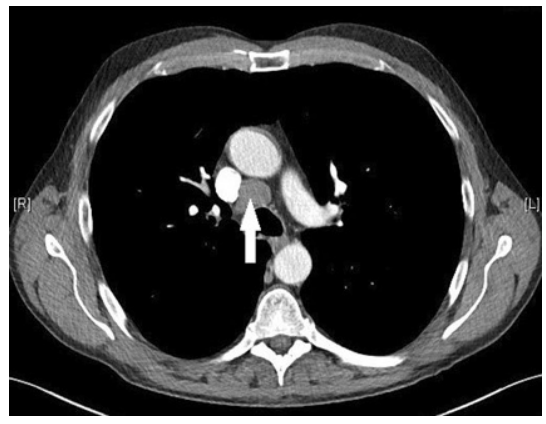

Abb. $5 \Delta$ Computertomographie des Thorax mit isoliertem paratrachealem auf $2,4 \mathrm{~cm}$ vergrößertem Lymphknoten (Pfeil)

prin, Cyclosporin, Mycophenolat-Mofetil, Rituximab oder Alemtuzumab beschrieben [3]. Ebenfalls liegen Fallberichte vor, die nach chirurgischer Entfernung des Primarius zu einer Visusverbesserung führten [5].

In der Mehrzahl der beschriebenen Fälle konnte durch die chirurgische, strahlen- und chemotherapeutische Behandlung des Primärtumors die visuelle Prognose lediglich stabilisiert, jedoch nicht verbessert werden. Ein weiterer therapeutischer Ansatz ist eine Reduktion der zirkulierenden Antikörper mittels Plasmapherese.

In dem von uns vorgestellten Fall lag zwischen okulären Erstsymptomen und Tumorsicherung eine Zeitspanne von 30 Tagen. Bereits der initiale Netzhautbefund zeigte ausgeprägte Destruktionen, welche sowohl unter der umgehend eingeleiteten Prednisolon-Therapie als auch der Plasmapherese zunahmen. Die Anamnese mit einem nur wenige Wochen vor Symptombeginn liegenden grippalen Infekt sowie die bestehende Panuveitis legten den Verdacht auf ein infektiöses bzw. parainfektiöses Geschehen nah. In der Thoraxröntgenaufnahme fand sich kein Hinweis auf den nur 2,4 cm großen Primärtumor, welcher erst durch das im Rahmen der Augensymptome angestoßene Screening detektiert werden konnte. Durch die eingeleitete Therapie konnte jedoch hochwahrscheinlich die Prognose für das Überleben des Patienten positiv beeinflusst werden.

\section{Fazit für die Praxis}

- Bei plötzlichen, unklaren Visusstö-
rungen bei anamnestisch gesundem 
In eigener Sache

Patienten auch an ein paraneoplastisches Geschehen denken.

- Augenärztliche Schlüsselposition bei der Einleitung und Koordinierung der Diagnose und interdisziplinären Therapie inklusive Antikörpernachweis und Tumorscreening.

\section{Korrespondenzadresse}

\section{Bettina Klieme}

Klinik und Poliklinik für Augenheilkunde, Martin-Luther-Universität Halle-Wittenberg Halle (Saale), Deutschland bettina.klieme@uk-halle.de

Funding. Open Access funding enabled and organized by Projekt DEAL.

\section{Einhaltung ethischer Richtlinien}

Interessenkonflikt. B. Klieme, A. Viestenz, S. Eisenmann und R. Wienrich geben an, dass kein Interessenkonflikt besteht.

Für diesen Beitrag wurden von den Autoren keine Studien an Menschen oder Tieren durchgeführt. Für die aufgeführten Studien gelten die jeweils dort angegebenen ethischen Richtlinien. Für Bildmaterial oder anderweitige Angaben innerhalb des Manuskripts, über die Patienten zu identifizieren sind, liegt von ihnen und/oder ihren gesetzlichen Vertretern eine schriftliche Einwilligung vor.

Open Access. Dieser Artikel wird unter der Creative Commons Namensnennung 4.0 International Lizenz veröffentlicht, welche die Nutzung, Vervielfältigung, Bearbeitung, Verbreitung und Wiedergabe in jeglichem Medium und Format erlaubt, sofern Sie den/die ursprünglichen Autor(en) und die Quelle ordnungsgemäß nennen, einen Link zur Creative Commons Lizenz beifügen und angeben, ob Änderungen vorgenommen wurden.

Die in diesem Artikel enthaltenen Bilder und sonstiges Drittmaterial unterliegen ebenfalls der genannten Creative Commons Lizenz, sofern sich aus der Abbildungslegende nichts anderes ergibt. Sofern das betreffende Material nicht unter der genannten Creative Commons Lizenz steht und die betreffende Handlung nicht nach gesetzlichen Vorschriften erlaubt ist, ist für die oben aufgeführten Weiterverwendungen des Materials die Einwilligung des jeweiligen Rechteinhabers einzuholen.

Weitere Details zur Lizenz entnehmen Sie bitte der Lizenzinformation auf http://creativecommons.org/ licenses/by/4.0/deed.de.

\section{Literatur}

1. Braithwaite T, Vugler A, Tufail A (2012) Autoimmune retinopathy. Ophthalmologica 228(3):131-142

2. Chan JW (2003) Paraneoplastic retinopathies and optic neuropathies. Surv Ophthalmol 48(1):12-38
3. Grewal DS, Fishman GA, Jampol LM (2014) Autoimmune retinopathy and antiretinal antibodies: a review. Retina 34(5):827-845

4. http://leitlinienprogramm-onkologie.de/ Lungenkarzinom. Zugegriffen: 18. Nov. 2020

5. Khattab M, Feltgen N, Bemme S, Hoerauf $H$, Storch M (2017) Bogenförmige retinale Aufhellung bei einer bisher gesunden Patientin. Opthalmologe 114:653-655

6. Ohguro H, Nakazawa M (2002) Pathological roles of recoverin in cancer-associated retinopathy. Adv ExpMed Biol 514:109-124

7. Sobottka B, Schlote T, Besch D, Djelebova T, Wilhelm H, Zrenner E (2000) Karzinomassoziierte Retinopathie: Eine Übersicht mit klinischen Beispielen. Klin Monbl Augenheilkd 216(1):17-24

\section{Bereich für Mitglieder der DOG auf SpringerMedizin.de}

Schauen Sie sich den neuen Bereich für Mitglieder der DOG auf SpringerMedizin.de an: www.springermedizin.de/dog

Hier finden Sie

- die Leitlinien, Empfehlungen und Stellungnahmen der DOG auf einen Blick,

- schnellen Zugriff auf die CME-Kurse von Die Ophthalmologie,

- das Online Archiv von Die Ophthalmologie rückwirkend bis 1997

- die aktuelle Ausgabe als e.Paper zum Offline-Lesen unterwegs,

- direkte Links zur Webseite der DOG und zum DOG E-Campus.

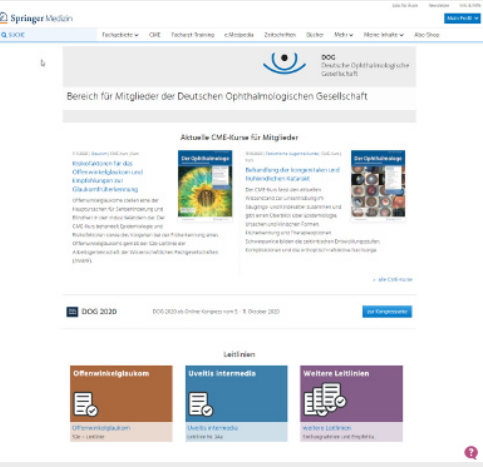

Registrieren Sie sich einmal kostenfrei auf www.springermedizin.de. Geben Sie dabei Ihren Vor- und Nachname und Lieferadresse wie auf dem Adressaufkleber auf Ihrem Heft an. So kann im System die Zugehörigkeit zur DOG sichergestellt werden.

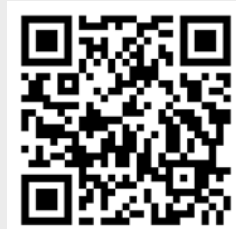

Einscannen und schnell und einfach lossurfen 